\title{
Assessing the quality of Smilacis Glabrae Rhizoma (Tufuling) by colormetrics and UPLC-Q-TOF-MS
}

Xicheng He ${ }^{1,2 \dagger}$, Tao Yi ${ }^{1 \dagger}$, Yina Tang ${ }^{1}$, Jun Xu' , Jianye Zhang ${ }^{1}$, Yazhou Zhang ${ }^{1}$, Lisha Dong ${ }^{2^{*}}$ and Hubiao Chen ${ }^{1^{*}}$

\begin{abstract}
Background: The quality of the materials used in Chinese medicine (CM) is generally assessed based on an analysis of their chemical components (e.g., chromatographic fingerprint analysis). However, there is a growing interest in the use of color metrics as an indicator of quality in CM. The aim of this study was to investigate the accuracy and feasibility of using color metrics and chemical fingerprint analysis to determine the quality of Smilacis Glabrae Rhizoma (Tufuling) (SGR). The SGR samples were divided into two categories based on their cross-sectional coloration, including red SGR (R-SGR) and white SGR (W-SGR).

Methods: Forty-three samples of SGR were collected and their colors were quantized based on an RGB color model using the Photoshop software. An ultra-performance liquid chromatography/quadrupole time-of-flight mass spectrometry (UPLC/QTOF MS) system was used for chromatographic fingerprint analysis to evaluate the quality of the different SGR samples. Hierarchical cluster analysis and dimensional reduction were used to evaluate the data generated from the different samples. Pearson correlation coefficient was used to evaluate the relationship between the color metrics and the chemical compositions of R-SGR and W-SGR.
\end{abstract}

Results: The SGR samples were divided into two different groups based on their cross-sectional color, including color $A(C L A)$ and $B(C L B)$, as well as being into two separate classes based on their chemical composition, including chemical $\mathrm{A}(\mathrm{CHA})$ and $\mathrm{B}(\mathrm{CHB})$. Standard fingerprint chromatograms were for $\mathrm{CHA}$ and $\mathrm{CHB}$. Statistical analysis revealed a significant correlation (Pearson's $r=-0.769, P<0.001$ ) between the color metrics and the results of the chemical fingerprint analysis.

Conclusions: The SGR samples were divided into two major clusters, and the variations in the colors of these samples reflected differences in the quality of the SGR material. Furthermore, we observed a statistically significant correlation between the color metrics and the quality of the SGR material.

Keywords: Smilacis Glabrae Rhizoma, Colormetrics, Fingerprint analysis

\section{Background}

The quality of Chinese medicine (CM) continues to attract considerable attention. The quality control procedures traditionally used in CM are based on the color, smell and texture characteristics of the raw materials, as

\footnotetext{
*Correspondence: dlsha1216@aliyun.com; hbchen@hkbu.edu.hk

${ }^{+}$Xicheng He and Tao Yi contributed equally to this work

${ }^{1}$ School of Chinese Medicine, Hong Kong Baptist Univesity,

Hong Kong, China

${ }^{2}$ School of Pharmacy, Gui Yang Collage of Traditional Chinese Medicine, Guiyang 550002, China
}

well as several other sensory properties. Color in particular is an essential method of quality control for evaluating the materials used in CM, but the effectiveness of this quality indictor is limited by its subjective nature. However, recent technological advances mean that it is now possible to measure and quantify colors using instrumental methods. For example, the major colors of an image can be represented by 8 bits in a computer, meaning that each color axis can be represented by 8 bits or $2^{8}=256$ different values in the Red-Green-Blue (RGB) color space $[1,2]$. Using imaging software, it is possible to read 
the RGB values of every pixel of a sample image. These data can subsequently be used to generate average RGB values for the different areas of an image, which can be used to represent color information.

Today, the quality of CM is mainly determined based on an analysis of their chemical composition using sophisticated analytical techniques, such as chromatographic fingerprint analysis, which can be used to provide an indication of their intrinsic quality. Instrument-based analytical techniques have therefore been widely accepted for quality evaluation and species identification in CM, supplementing traditional techniques based on the sensory properties of these materials [3-5]. Establishing a connection between the color and the chemical composition of the materials used in CM would provide solid evidence for establishing a new method of quality control, which could validate the correlation between the sensory properties and quality of CM. In this study, we have selected Smilacis Glabrae Rhizoma (Tufuling) (SGR) as a model medicinal herb to evaluate the relationship between the quality of this material and its color metrics and chemical composition data.

SGR, which is the dried rhizome of Smilax glabra Roxb., can be red or white (Fig. 1). Generally, the therapeutic effects of white SGR are believed to be more pronounced than those of the red material [6-8], suggesting that the color of this material could be used as an indicator of its quality. SGR samples can therefore be divided into two groups depending on their cross-sectional color, including red SGR (R-SGR) and white SGR (W-SGR) [6].

However, the results of another study revealed that most SGR samples contain both red and white crosssections, and that the quality of these materials can vary considerably [9]. Despite the potential implications of this discovery, there has been other work conducted in this area, and the quality of SGR is currently evaluated by chemical composition analysis with no regard for differences in the apparent color.

This aim of this study was to investigate the accuracy and feasibility of using color metrics and chemical fingerprint analysis to analyze the quality of SGR. In this study, we used image software to quantify the color information of the red and white SGR samples based on color data generated using an RGB model. We also used ultraperformance liquid chromatography/quadrupole time-offlight mass spectrometry (UPLC/QTOF MS) to produce chemical chromatographic fingerprints for these different samples. Hierarchical analysis and dimensional reduction methods were used to generate color and chemical scores for the different SGR samples, and standard fingerprint chromatograms were established according to the clustering results of the main model. Pearson correlation coefficient was used to evaluate the relationship between the color and the chemical composition of the different samples, which revealed a statistically significant relationship between these two variables (Fig. 2a).

\section{Methods}

\section{Materials}

Information pertaining to the different SGR samples is shown in Table 1. All of these samples were authenticated as the genuine rhizome of S. Glabra by Prof. Lisha Dong (School of Pharmacy, Gui Yang College of Traditional Chinese Medicine, China). These samples were authenticated based on a comparison of their flowers with those of Smilax Glabra Roxb., according to the Flora of China [10].

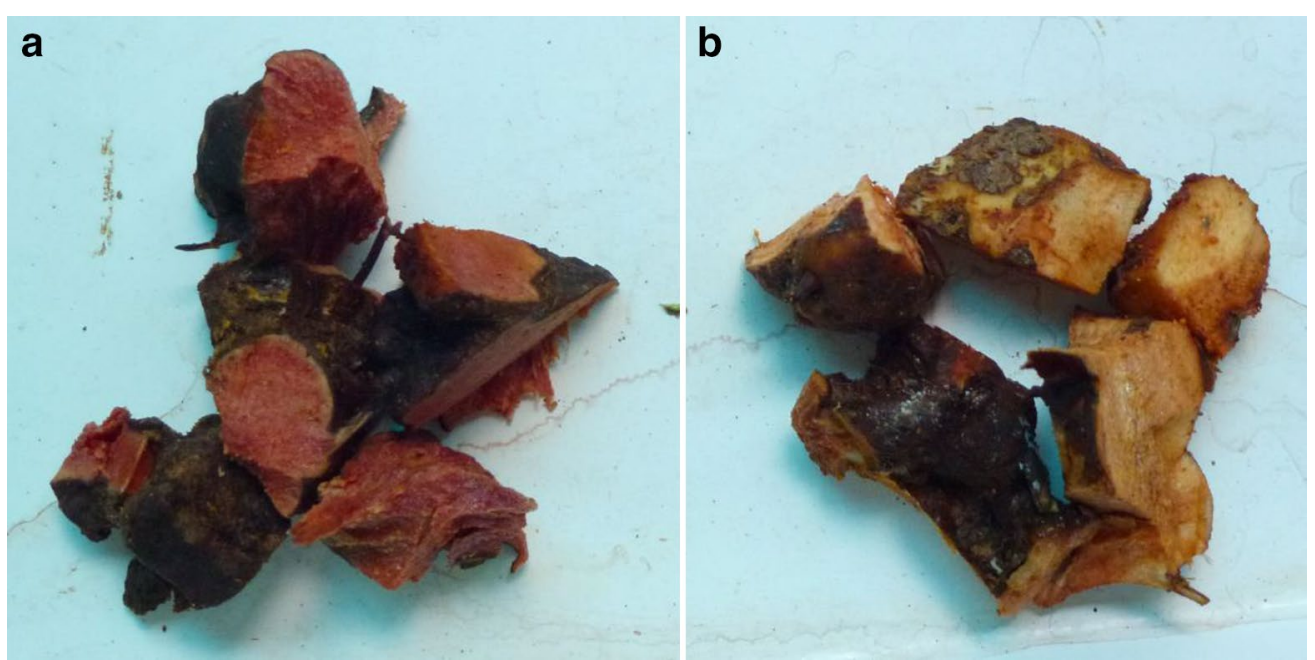

Fig. 1 The samples of SGR (a) Red cross-sectional SGR. b White cross-sectional SGR 
a

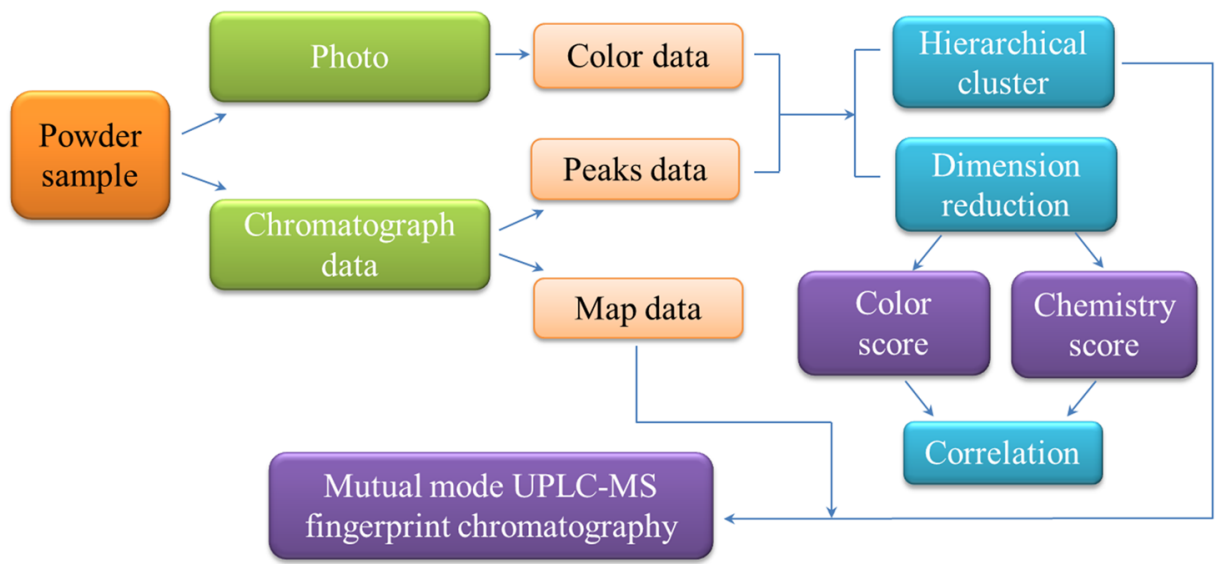

b

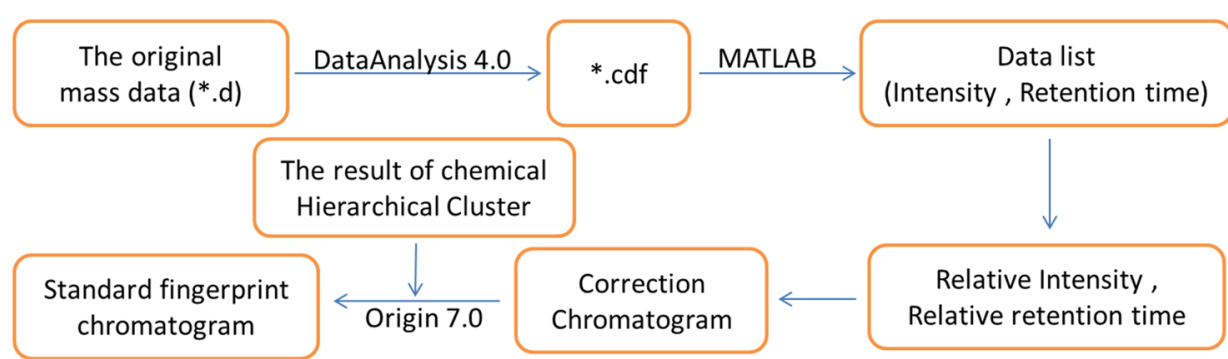

Fig. 2 Flow chart of this work. a Flow chart of the experiment design process (b) Flow chart for establishing a standard fingerprint chromatogram RIntens. $=\frac{\text { Intens. }}{\mathrm{SPH} \times \mathrm{W}}, \quad R R T=\frac{\mathrm{RT}}{\mathrm{SRT}}$, where $R$ Intens is the relative Intensity, $R \mathrm{RT}$ is the relative retention time, SPH and SRT are the peak height and retention time, respectively, of the internal standard (IS), and W was sample weight (g)

Analytical grade methanol (Labscan, Bangkok, Thailand) was used to prepare the reference standards, as well as the samples of the different extracts. Chromatography-grade formic acid (Fluka, Buchs, Switzerland), chromatography-grade acetonitrile (Labscan, Bangkok, Thailand) and Milli-Q water (Millipore, Bedford, MA, USA) were used to prepare the mobile phases for chromatography. Analytical standards of astilbin, engeletin, (+)-catechin, (-)-epicatechin and 7,4'-dihydroxyflavone were obtained from the National Institute for the Control of Pharmaceutical and Biological Products, China. All of these chemical standards were greater than $98 \%$ in purity.

\section{Analytical procedures Color analysis}

The SGR samples were pulverized, and the resulting powders were sieved through a 120-mesh screen. The sieved powders were then stored in the dark prior to being analyzed. Samples of the sieved powders were uniformly dispersed on the surface of a glass culture dish (diameter, $60 \mathrm{~mm}$ ) which was placed in a Desktop Proeasy LTM-101 light source system (Nexor, Guangzhou, China) together with a standard color checker (Mennon, Beijing, China) and an $18 \%$ Grey Card (Topimage, Taipei, Taiwan). Photographic images were recorded using a Nikon D5200 camera with a focal length of $24 \mathrm{~mm}$ (M grade, A:F8, $\mathrm{S}: 1 / 40$ ). The photographic images were visualized using the Adobe Photoshop CS5 software (Adobe, San Jose, CA, USA). The Grey Card was used to correct the white balance in the images using the gray-pipette tool from Photoshop, whereas the standard color checker was used to produce a file containing less than five color errors. Five different areas of each photograph were selected for sampling, with each sampling area set as $51 \times 51$ pixels. Color data were obtained by calculating the average values of the RGB data, which were measured using the color information derived from each sample with the eyedropper tool from Photoshop (Fig. 3).

Color data were subjected to partial least squares (PLS) regression analysis using the MATLAB software (MathWorks, Natick, MA, USA) to analyze the relationship between the colors in R-SGR and W-SGR, as well as quantifying the boundary between R-SGR and W-SGR. The dependent variable used in this model was $\mathrm{G}$, whereas $\mathrm{R}$ and $\mathrm{B}$ were used as independent variables. 
Table 1 Details of the different SGR samples

\begin{tabular}{|c|c|c|c|}
\hline No. & Batch no. & Location & $\begin{array}{l}\text { Color of } \\
\text { cross-section }\end{array}$ \\
\hline 1 & 20080728 & Guiyang, Guizhou & Red \\
\hline 2 & 20090403 & Guiyang, Guizhou & Red \\
\hline 3 & 20090828 & Taizhou, Zhejiang & White \\
\hline $4^{a}$ & 20090922 & Beijing Tongrentang & White \\
\hline $5^{a}$ & 20090928 & Luoyang, Henan & White \\
\hline 6 & 20100316 & Guangdong & White \\
\hline 7 & 20100410 & Guizhou & White \\
\hline $8^{a}$ & 20100429 & Sichuan & White \\
\hline 9 & 20100513 & Liubanshui, Guizhou & Red \\
\hline 10 & 20100531 & Guiyang, Guizhou & Red \\
\hline 11 & 20100606 & Guiyang, Guizhou & White \\
\hline 12 & 20100704 & Anshun, Guizhou & Red \\
\hline $13^{\mathrm{a}}$ & 20100714 & Anhui & White \\
\hline 14 & 20100715 & Guiyang, Guizhou & Red \\
\hline 15 & 20100821 & Guiyang, Guizhou & Red \\
\hline 16 & 20100826 & Guiyang, Guizhou & White \\
\hline 17 & 20100901 & Guiyang, Guizhou & White \\
\hline 18 & 20100924 & Guiyang, Guizhou & Red \\
\hline 19 & 20101028 & Tongren, Guizhou & White \\
\hline 20 & 20100104 & Zhijin, Guizhou & Red \\
\hline 21 & 20101005 & Duyun, Guizhou & Red \\
\hline 22 & 20101111 & Zunyi, Guizhou & White \\
\hline $23^{a}$ & 101201 & Guiyang Tongrentang & White \\
\hline 24 & 20110210 & Zhijin, Guizhou & Red \\
\hline $25^{a}$ & 110410 & Guiyang Tongrentang & White \\
\hline 26 & 20110715 & Duyun, Guizhou & Red \\
\hline $27^{\mathrm{a}}$ & 20110601 & Anhui & White \\
\hline 28 & 20110805 & Guiyang, Guizhou & Red \\
\hline 29 & 20100812 & Guiyang, Guizhou & Red \\
\hline 30 & 20110831 & Guiyang, Guizhou & Red \\
\hline 31 & 20110903 & Guiyang, Guizhou & Red \\
\hline $32^{\mathrm{a}}$ & 20111005 & Hangzhou, Zhejiang & White \\
\hline 33 & 20110318 & Puan, Guizhou & Red \\
\hline 34 & 20120415 & Hezhang, Guizhou & Red \\
\hline 35 & 20120714 & Ceheng, Guizhou & White \\
\hline 36 & 20130104 & Hongkong & White \\
\hline 37 & 20120402 & Puan, Guizhou & Red \\
\hline 38 & 20120418 & Duyun, Guizhou & White \\
\hline 39 & 20121220 & Hong Kong & White \\
\hline 40 & 20130214 & Qingyuan, Guangdong & White \\
\hline 41 & 20130530 & Baise, Guangxi & Red \\
\hline 42 & 20130626 & Nanning, Guangxi & White \\
\hline 43 & 20130628 & Wuzhou, Guangxi & White \\
\hline
\end{tabular}

${ }^{a}$ Represent purchased samples

\section{Chemical analysis}

An SGR power sample of $0.5 \mathrm{~g}$ was added to $20.0 \mathrm{~mL}$ of $60 \%(\mathrm{v} / \mathrm{v})$ methanol in water, and the resulting mixture

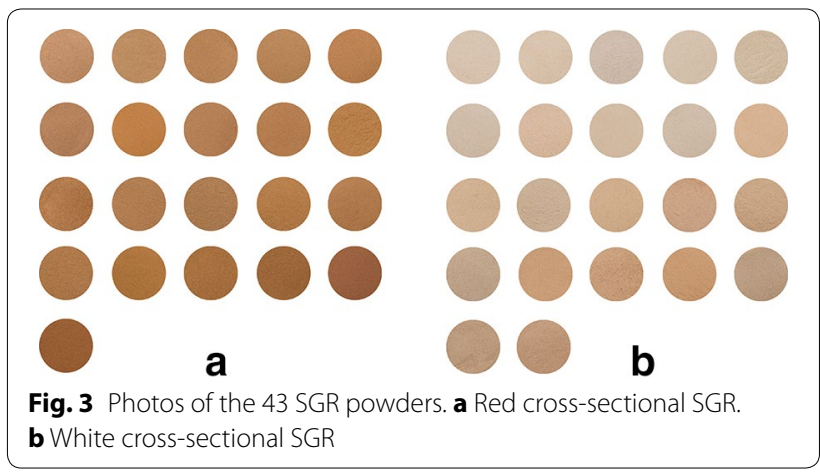

was sonicated (CP1800 HT, Crest, Penang-, Malaysia) for $30 \mathrm{~min}$ at room temperature. The sonicated mixture was centrifuged (5810, Eppendorf, Hamburg, Germany) at $2100 \times g$ for $5 \mathrm{~min}$ at room temperature, and $400 \mu \mathrm{L}$ of the supernatant was placed in a volumetric flask, followed by $100 \mu \mathrm{L}$ of $7,4^{\prime}$-dihydroxyflavone $(0.1249 \mathrm{mg} / \mathrm{mL})$, which was added as an internal standard (IS). The total volume of the mixture in the volumetric flask was then adjusted to $10 \mathrm{~mL}$ by the addition of methanol, and the resulting sample solution was filtered through a $0.22-\mu \mathrm{m}$ filter before being analyzed by LC-MS. Two replicates of each SGR sample were prepared and analyzed in the same way. All of the extracts were stored at $4{ }^{\circ} \mathrm{C}$ before use.

UPLC analysis was performed on a Waters Acquity system (Waters Corporation, Milford, MA, USA) equipped with a quaternary solvent manager and a sample manager. This system was also coupled to a Micromass QTOF premier mass spectrometer (Bruker Daltonics, Milford, MA, USA) equipped with an electrospray ionization (ESI) interface. Chromatographic separation was conducted over a Waters C18 T3 column $(1.8 \mu \mathrm{m}, 2.1 \times 100 \mathrm{~mm})$ eluting with a mobile phase consisting of $0.1 \%$ formic acid in water (A) and $0.1 \%$ formic acid in acetonitrile (B) with linear gradient elution at a flow rate of $0.35 \mathrm{~mL} / \mathrm{min}$. The gradient elution was conducted as follows: $5-14 \% \mathrm{~B}$ (0-1.5 min), 14-16 \% B (1.5-6.0 min), 16-20\% B (6.0$20.0 \mathrm{~min}), 20-100 \% \mathrm{~B}(20.0-30.0 \mathrm{~min})$. The column temperature was kept at $40{ }^{\circ} \mathrm{C}$ throughout the entire process. The sample size for injection into the UPLC system was set at $2 \mu \mathrm{L}$. The ESI source of the MS was connected to the UPLC system via a capillary through the UV cell outlet. MS data were collected with a capillary voltage of $3.5 \mathrm{kV}$ in the negative ionization mode. Nitrogen was used as a desolvation gas at a flow rate was of $8 \mathrm{~L} / \mathrm{h}$. The scan range $(\mathrm{m} / \mathrm{z})$ was set at $100-1000 \mathrm{Da}$.

Extracted ion chromatograms (EIC) were used to obtain the peak areas of the different components, and the relative peak areas were then calculated based on the peak area of the IS. The relative peak areas were calculated using the following equation: 


$$
R P A_{i}=\frac{A_{i}}{A_{S} \times W},
$$

where $R P A$ is the relative peak area, $A$ is the peak area of a specific component, $A_{s}$ is the peak area of the IS, W is the sample weight and $i$ is the peak number.

The original data were saved as CDF files using version 4.0 of the DataAnalysis software (Bruker, USA). The MATlab R2012b software (MathWorks, USA) was then used to extract information from the CDF files, including retention time (RT) and intensity (Intens.) data (Fig. 2b). The scan range $(\mathrm{m} / \mathrm{z})$ was set at $100-800 \mathrm{Da}$. According to the result of chemical hierarchical cluster, chromatogram maps corrected by IS were combined to generate standard chromatogram by mean value method.

The correlation coefficient, $r_{c o n}$, was used in the current study to assess the consistency of the chemical composition of SGR using the following equations $[3,11,12]$ :

$$
\begin{aligned}
& r_{\text {con }}=\frac{\sum_{i=1}^{n u m} x_{i} y_{i}}{\sqrt{\left(\sum_{i=1}^{\text {num }}\left(x_{i}\right)^{2}\right)\left(\sum_{i=1}^{\text {num }}\left(y_{i}\right)^{2}\right)}}, \\
& \bar{x}=\left(\sum_{i=1}^{n u m} x_{i}\right) / n, \quad \bar{y}=\left(\sum_{i=1}^{n u m} y_{i}\right) / n,
\end{aligned}
$$

where $x_{i}$ and $y_{i}$ are the $i$ th elements in two different fingerprints (i.e., $x$ and $y$ ), num is the number of the elements in the fingerprints and $\bar{x}$ and $\bar{y}$ are the mean values of the num elements in fingerprints $x$ and $y$, respectively.

\section{Correlation of color metrics and chemical fingerprint}

Dimensional reduction was used to convert complex chemical information and color data into one or a few simple variables. Factor analysis was used to reduce the number of dimensions and extract the most important information from the original data. To verify that the resulting data sets were suitable for factor analysis, we checked that the Kaiser-Meyer-Olkin (KMO) values were greater than 0.6 and that the Bartlett's test of Sphericity value was significant (significant values should be less than 0.05). The color and chemical scores were subsequently calculated using factor analysis, and the results were used to evaluate the correlation between the color metrics and the chemical fingerprint by Pearson correlation coefficient.

\section{Statistical analysis \\ Hierarchical clustering}

Hierarchical clustering was conducted using version 20.0 of the IBM SPSS software (IBM, Armonk, NY, USA) to allow for the standardization of the color and chemical data, followed by hierarchical clustering by classification analysis.

\section{Dimensional reduction}

Factor analysis was used for the dimensional reduction of the color data obtained for the different SGR samples, and the resulting factor score was subsequently regarded as the color score. Factor analysis was used for dimensional reduction processing, and the principal components were considered to be independent based on the factor rotation method. Based on the different values of the principal component analysis, we calculated a cumulative score for the chemical data. $P$ value less than 0.05 were considered statistically significant.

\section{Correlation analysis}

The relationship between the color and chemical scores was evaluated using Pearson correlation coefficient. A scatter plot of these data was then generated using version 20.0 of the SPSS software with the chemical and color scores on the $\mathrm{x}$ and $\mathrm{y}$ axes, respectively. $P$ value less than 0.05 were considered statistically significant.

\section{Results and discussion Color analysis}

A color dendrogram of the 43 different SGR samples evaluated in this study is shown in Fig. 4a. This figure shows that the SGR samples were divided into two major clusters (i.e., R-SGR and W-SGR) based on their color. The R-SGR samples were grouped into one cluster defined as "CLA", whereas the W-SGR samples were grouped into another cluster defined as "CLB". The results of quantitative color analysis supported the result of this subjective classification, suggesting that SGR samples were composed of both white and red materials. However, it is noteworthy that all of these samples were defined as being the same based on their original botanical identification.

The major colors of the different SGR samples were represented by one of 256 different values in the RGB color space. Based on the results of the regression analysis, we established a multiple regression model in color space using the following regression equation (Fig. 4c):

$$
G=-17.18+0.62 R+0.46 B\left(R^{2}=0.998 ; P<0.001\right) .
$$

All of the R-SGR and W-SGR samples were represented in this regression model. As shown in the expanded view, this model established a standard tape of SGR, with R-SGR and W-SGR on either side based on the color location, allowing for the two clusters to be differentiated into two different zones. 


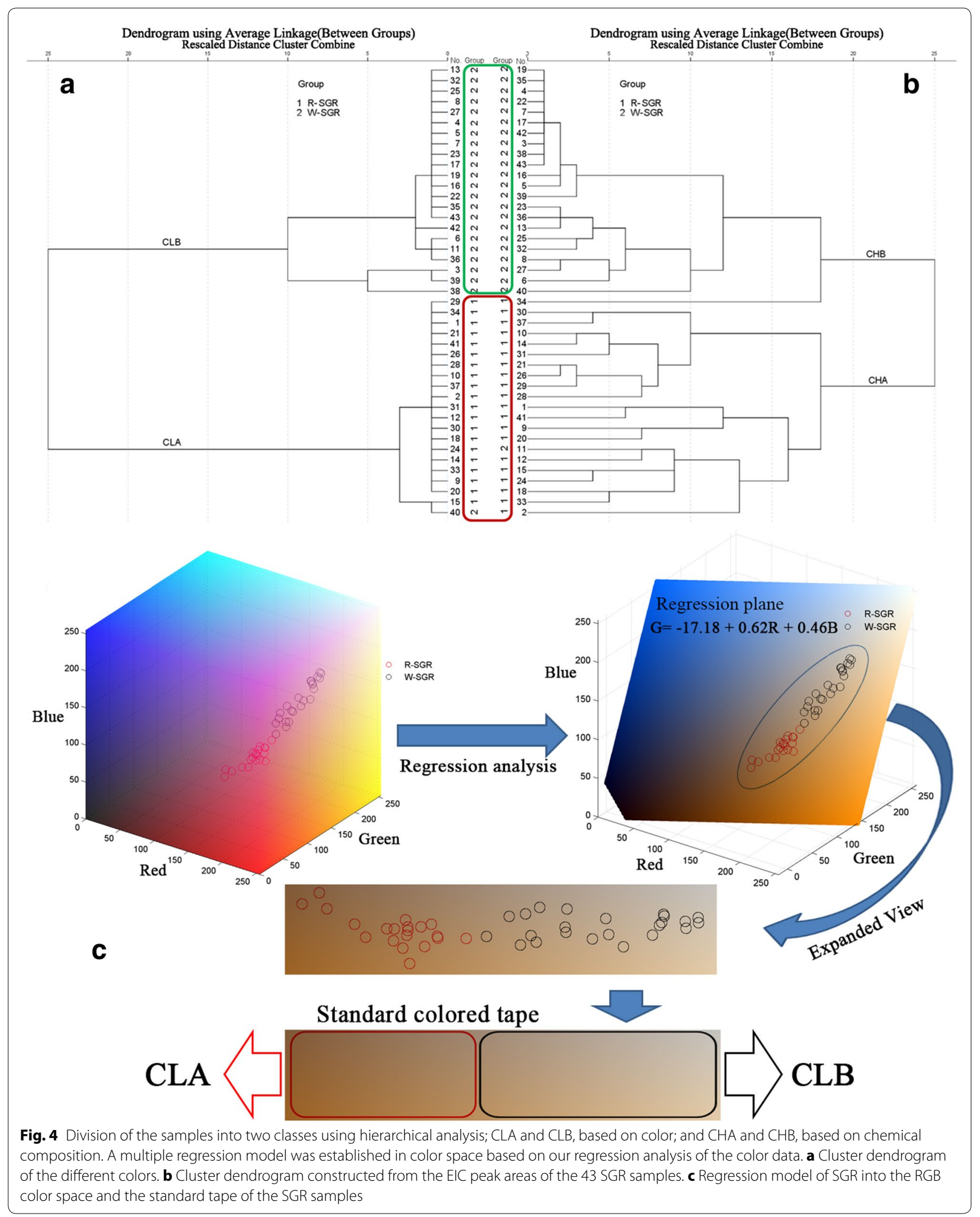




\section{Chemical analysis}

The 43 SGR samples were also subjected to chemical analysis and separated into two categories based on the results by hierarchical clustering (Fig. 4b). The R-SGR samples were grouped in one cluster, which was defined as "CHA", whereas the W-SGR samples were grouped in anther cluster, which was defined as "CHB", indicating that there were two different types of SGR based on differences in their chemical composition.

The chemical compositions in the extracts of R-SGR and W-SGR were analyzed by UPLC/QTOF MS. Twentyeight characteristic peaks were found in the EICs and nine compounds were identified based on their fragmentation patterns and a comparison of their MS data with those available in the literature (Table 2) [13, 14, 15]. The R-SGR and W-SGR samples contained different numbers of peaks (compositions), as shown in Fig. 5a, b.

Table 2 Information concerning the different compounds found in the chromatogram of SGR

\begin{tabular}{|c|c|c|c|}
\hline No. & RRT & $\mathrm{m} / \mathrm{z}(-)$ & Compounds \\
\hline 1 & 0.106 & 323.13 & \\
\hline 2 & 0.174 & 289.07 & $(+)$-Catechin \\
\hline 3 & 0.180 & 561.14 & \\
\hline 4 & 0.211 & 561.14 & \\
\hline 5 & 0.230 & 289.07 & (-)-Epicatechin \\
\hline 6 & 0.236 & 561.14 & \\
\hline $7^{*}$ & 0.255 & $335.07 / 179.04$ & 5-O-Caffeoylshikimic acid \\
\hline 8 & 0.261 & 561.14 & \\
\hline 9 & 0.280 & 465.10 & \\
\hline 10 & 0.304 & 339.07 & \\
\hline 11 & 0.329 & $335.07 / 179.04$ & \\
\hline 12 & 0.354 & $449.08 / 269.05$ & \\
\hline 13 & 0.391 & 449.08 & \\
\hline 14 & 0.422 & $451.10 / 341.06$ & \\
\hline 15 & 0.435 & 507.11 & \\
\hline $16^{*}$ & 0.453 & $449.08 / 303.05$ & Neoastilbin \\
\hline 17 & 0.472 & $449.08 / 269.05$ & \\
\hline 18 & 0.491 & $449.08 / 303.05$ & \\
\hline 19 & 0.534 & 723.17 & \\
\hline $20^{*}$ & 0.609 & $449.08 / 303.05$ & Astilbin \\
\hline 21 & 0.634 & 491.11 & \\
\hline $22^{*}$ & 0.652 & $449.08 / 303.05$ & Isoastilbin \\
\hline 23 & 0.702 & $433.1 / 269.04$ & Engeletin \\
\hline 24 & 0.795 & 723.50 & \\
\hline 25 & 0.876 & 491.11 & \\
\hline 26 & 0.944 & 491.11 & \\
\hline $27^{*}$ & 0.969 & $433.11 / 269.04$ & Isoengeletin \\
\hline IS & 1.000 & 253.05 & 7,4'-di Hydroxy flavone \\
\hline 28 & 1.106 & 451.10 & \\
\hline
\end{tabular}

*Represent that the peak was identified by references
The amounts of the different components also changed between the two different sample types.

The precision of this analytical method was determined by injecting the same sample solution into the system on six consecutive occasions. The peak areas of the different components were then taken as measures of the precision and expressed as relative standard deviation (RSD) values, which resulted in a precision of $2.97 \%$. Six peaks with peak areas greater than $3 \%$ and relative retention time (RRT) of $0.25,0.35,0.45,0.49,0.65$ and 0.70 were chosen to calculate the repeatability and stability of the method. The RSD of RT repeatability was less than $0.24 \%$, whereas the relative peak area (RPA) repeatability was less than $4.72 \%$, which showed good stability over $8 \mathrm{~h}$ at less than $4.57 \%$.

Standard fingerprint chromatograms of samples belonging to the $\mathrm{CHA}$ and $\mathrm{CHB}$ groups were established based on the result of the main clustering model (Fig. 5a, b), and calculated with a $95 \%$ confidence interval. A pronounced difference was observed between the two modes of examination used in the standard fingerprint chromatograms. The standard fingerprint chromatograms of CHA contained 28 peaks, whereas standard fingerprint chromatograms of CHB contained only 17 peaks without peak numbers 12, 17, 21, 25 and 26. Moreover, the amount of the different components varied considerably between $\mathrm{CHA}$ and $\mathrm{CHB}$. For example, the peak corresponding to astilbin (peak 18), which was the biggest of all of the peaks detected in $\mathrm{CHA}$ and $\mathrm{CHB}$, was much smaller in $\mathrm{CHB}$ than it was in CHA. Five other peaks, including peaks 2, $5,7,22$ and 23, also appeared at much lower levels in the standard fingerprint in CHB.

The relationships between the fingerprint chromatograms could be analyzed by comparing the similarities between specific reference points. The correlation coefficient was used in this study to examine the similarities between the samples clustered into the CHA and $\mathrm{CHB}$ groups. The correlation coefficients of the $\mathrm{CHA}$ were more than 0.9 (the red line in Fig. 5c), indicating that the standard fingerprint chromatograms represented the chemical characteristics of R-SGR. All of the correlation coefficients of the CHB were greater than 0.93 (the blue line in Fig. $5 \mathrm{~d}$ ), and the similarities in this group were higher than those of the CHA group, indicating that the standard fingerprint chromatograms represented the chemical characteristics of W-SGR. The standard fingerprint chromatograms of $\mathrm{CHA}$ and $\mathrm{CHB}$ were stable and consistent.

\section{Correlation of the color metrics and chemical fingerprint data}

For this example, we obtained a KMO value of 0.656 , and the results of a Bartlett's test revealed that this result was significant $(P<0.001)$, indicating that the use of factor analysis 


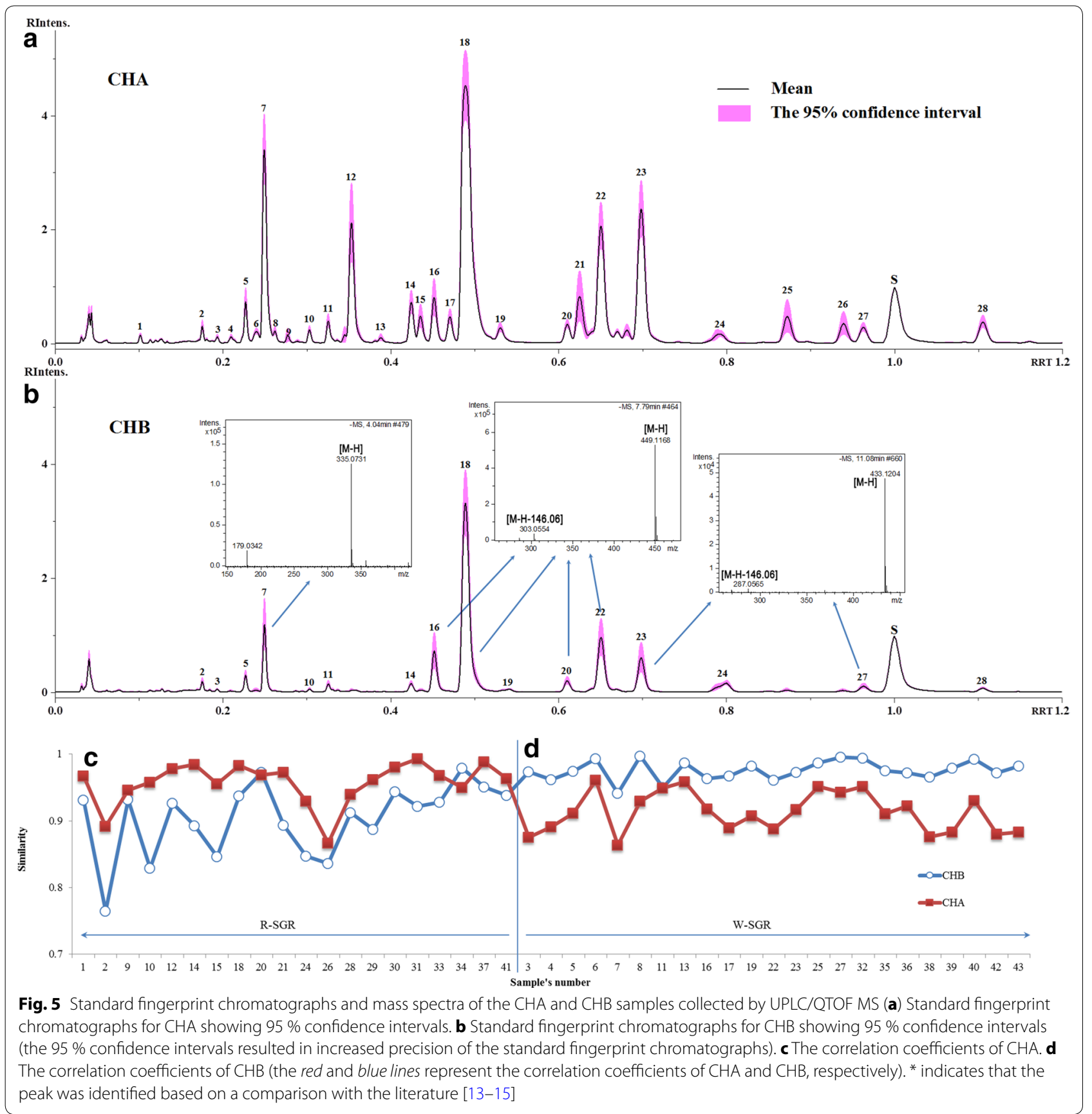

was appropriate [16]. The three variables (RGB) for the color data were transformed into one factor score, affording a total cumulative variance of $97.851 \%$. The color score was calculated as follows:

Color score $=Z R \times 0.334+Z G \times 0.340+Z B \times 0.337$,

where $Z R, Z G$ and $Z B$ are standardized data for $R, G$ and $B$, respectively. The constants were defined as the component score coefficients. A high color score was indicative of white SGR, whereas a low color score was indicative of red SGR.
The results of the chemical data led to the identification of six principal factors based on 28 peaks by factor analysis. Principal component analysis was then used to translate these six variables into a chemical score, which gave a total cumulative variance of $88.298 \%$ together with a KMO value of 0.639. Furthermore, Bartlett's test was significant $(P<0.001)$. The formula used to compute the chemical score was as follows: Chemistry score $=$ FAS1 $\times 20.947 \%+$ FAS $2 \times 16.818 \%+$ FAS3 $\times 16.702 \%+$ FA S $4 \times 15.418 \%+$ FAS5 $\times 13.717 \%+$ FAS6 $\times 4.696 \%$. 


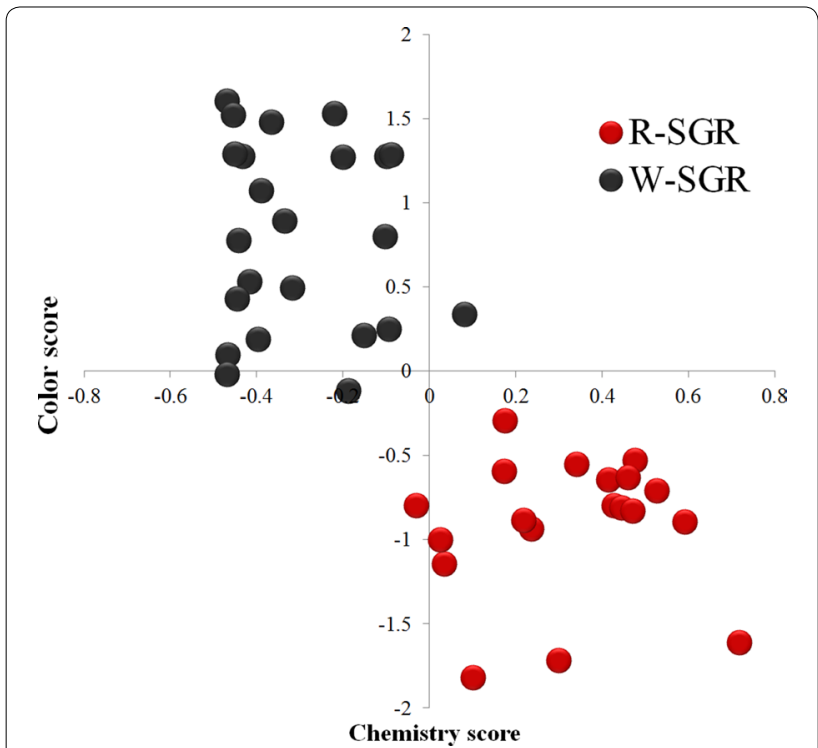

Fig. 6 Scatter diagram of the color and chemistry scores

FAS was used as a factor score, with constant squared loadings for each factor. This method allowed for the evaluation of increasingly complex factors, and also provided a novel strategy for evaluating quality.

The Pearson correlation coefficient was determined to be -0.769 (double side test $P<0.001$ ), suggesting that there was a significant correlation between the color and chemistry scores (Fig. 6).

The result of the dimensional reduction and correlation analysis indicated that there was a statistically significant relationship between the colors and chemical components of the different SGR samples, as did the results of the hierarchical clustering. These results therefore suggest that it is feasible to use color (red and white) to distinguish between two different qualities or specifications of SGR.

\section{Conclusions}

SGR samples were divided into two major clusters and the variation in their color provided a good indication of the differences in their quality. Notably, we observed a statistically significant correlation between the color metrics and the quality of the different SGR samples (Pearson's $\mathrm{r}=-0.769, P<0.001$ ).

\section{Abbreviations}

CM: Chinese medicine; SGR: Smilacis Glabrae Rhizoma; R-SGR: red SGR; W-SGR: white SGR; RGB: Red-Green-Blue; UPLC-Q-TOF-MS: ultra-performance liquid chromatography-quadrupole time-of-flight mass spectrometry; CLA: color $\mathrm{A}_{\text {; }}$ CLB: color B; CHA: chemical A; CHB: chemical B; ESI: electrospray ionization; EIC: extracted ion chromatogram; RPA: relative peak area; $R T$ : retention time; IS: internal standard; KMO: Kaiser-Meyer-Olkin; RSD: relative standard deviation; $\mathrm{RRT}$ : relative retention time.

\section{Authors' contributions}

HC, LD, XH and TY designed the study. XH, TY and YT performed the experiments, $T Y$ and $X H$ analyzed the data. $X H, T Y, J X, J Z$ and $Y Z$ wrote the manuscript. All authors read and approved the final manuscript.

\section{Acknowledgements}

This study was partially supported by grants from the National Natural Science Foundation of China (Project codes: 30960503 and 81460588) and QianKeHe ZY Zi of the Development of Guizhou Science and Technology Agency, China (Project No. 30011). We would also like to thank Ho Hing Man and Yeung Wing Ping for providing technical support.

\section{Competing interests}

The authors declare that they have no competing interests.

Received: 16 April 2014 Accepted: 21 June 2016

Published online: 06 July 2016

\section{References}

1. Balaban MO. Quantifying nonhomogeneous colors in agricultural materials part I: method development. J Food Sci. 2008;73:S431-7.

2. Balaban MO, Aparicio J, Zotarelli M, Sims C. Quantifying nonhomogeneous colors in agricultural materials. Part II: comparison of machine vision and sensory panel evaluations. J Food Sci. 2008;73:S438-42.

3. Zhu HB, Wang CY, Qi Y, Song FG, Liu ZQ, Liu SY. Fingerprint analysis of Radix Aconiti using ultra-performance liquid chromatography-electrospray ionization/tandem mass spectrometry (UPLC-ESI/MSn) combined with stoichiometry. Talanta. 2013;103:1-10.

4. Liu ZL, Liu YY, Wang C, Guo N, Song ZQ, Wang C, Xia L, Lu AP. Comparative analyses of chromatographic fingerprints of the roots of Polygonum multiflorum Thunb. and their processed products using RRLC/DAD/ESIMSn. Planta Med. 2011;77:1855-60.

5. Xie GX, Ni Y, Su MM, Zhang YY, Zhao AH, Gao XF, Liu Z, Xiao PG, Jia W. Application of ultra-performance LC-TOF MS metabolite profiling techniques to the analysis of medicinal Panax herbs. Metabolomics. 2008:4:248-60

6. Jiang RL, Liu HM, Chen GR, Wang JS, Wu HZ, Chen WQ, Tang SP, Xia DQ, Zhong $\mathrm{DH}$. Changyong zhongyaocai pingzhongzhengli he zhiliangyanjiu tufulinlei zhuanti yanjiu. SiChuan zhongcaoyao yanjiu. 1992;33:1-17.

7. Xie ZW. Zhongyao pinzhong lilun yu yingyong. 1st ed. Beijing: People's Medical Publishing House; 2008. p. 490-4.

8. Xie ZW. Zhongyaocai pinzhong lunsu. 2nd ed. Shanghai: Scientific and Technical Publishers; 1999.

9. He XC, Shun QW, Ge XQ, Zhang H, Nong H, Shen XH, Dong LS. Comparison of anti-inflammatory effect and analysis of astilbin red and white transverse section Smilax glabra Roxb. in 28 collection sites. Zhongguo zhongyao zazhi. 2012;37:3593-8.

10. Tang J, Wang FZ. Flora of China, vol. 15th. Beijing: Science Press; 2004. p. 212.

11. Xu CJ, Liang YZ, Chau FT, Vander HY. Pretreatments of chromatographic fingerprints for quality control of herbal medicines. J Chromatogr A 2006;1134:253-9.

12. Lu HM, Liang YZ, Chen S. Identification and quality assessment of Houttuynia cordata injection using GC-MS fingerprint: a standardization approach. J Ethnopharmacol. 2006;105:436-40.

13. Zhang QF, Cheung HY, Zeng LB. Development of HPLC fingerprint for species differentiation and quality assessment of Rhizoma Smilacis Glabrae. J Nat Med. 2013;67:207-11.

14. Wang YH, Li L, Zhang HG, Qiao YJ. Identification of dihydroflavonol glycoside isomers in Smilax glabra by HPLC-MS and HPLC-'H NMR. Zhongguo zhongyao zazhi. 2008;33:1281-4.

15. Chen L, Yin Y, Yi HW, Xu Q, Chen T. Simultaneous quantification of five major bioactive flavonoids in Rhizoma smilacis glabrae by high-performance liquid chromatography. J Pharm Biomed Anal. 2007:43:1715-20.

16. Julie P. SPSS Survival Manual: a step by step guide to data analysis using SPSS for windows. 3rd ed. New York: Open University Press; 2007. 